\title{
6. Ergebnisse
}

In der vorliegenden Arbeit wurde die Verteidigungsanlage von Konstantinopel im Westen der Stadt vom Marmarameer bis zum Goldenen Horn unter verschiedenen Aspekten untersucht. Die dabei gewonnenen Erkenntnisse sollen im folgenden kurz zusammengefaßt werden:

1. Die bisher vorherrschende Meinung, daß die Theodosianische Landmauer auf der Höhe von Tekfur Sarayı an einen alten Mauerabschnitt anschloß und am Endpunkt dieser Mauer unter Kaiser Theodosius II. ein Zweifronten-Wehrgang (das sog. Pteron) erbaut worden sei, scheint nicht haltbar zu sein, da die Mauer nördlich Tekfur Sarayı ihrem Mauerwerk nach nicht früher als die theodosianische Mauer erbaut worden sein kann und möglicherweise erst zur Zeit des Kaisers Herakleios errichtet wurde. Die theodosianische Mauer muß im ursprünglichen Zustand einen ganz anderen Verlauf genommen haben und von Tekfur Sarayı nach Nordosten abbiegend mit einem Mauerrest wenig südlich der Demetrios-Kirche ó Kaváßฤৎ zusammengetroffen sein.

2. Die angenommene Anzahl der Türme - 96 - entspricht nicht dem theodosianischen Konzept. Die Türme 83, 85 und 88 waren zur Zeit Theodosius' II. nicht mitgeplant worden. Ihre Anbringungsorte sowie einige architektonische Besonderheiten sprechen vielmehr dafür, daß es sich dabei um spätere Scheintürme handelt. Wie auch das Mauerwerk erkennen läßt, sind diese in die mittelbyzantinische Zeit (9. bzw. 10. Jahrhundert) zu datieren. Des weiteren kann auch die ursprüngliche Existenz von Turm 39 bezweifelt werden. Dagegen könnte sich an der Stelle des heutigen Vorturms 40a ein theodosianischer Hauptturm befunden haben. Im ursprünglichen Zustand muß es zwischen Marmarameer und Tekfur Sarayı mindestens 94 und höchstens 95 Türme gegeben haben.

3. Es ist sicher davon auszugehen, daß Haupt- und Vormauer des theodosianischen Bauabschnitts vor dem Jahr 425 gleichzeitig konzipiert und auch gleichzeitig oder kurz hintereinander errichtet worden sind. Die immer wieder in die Debatte eingebrachte Meinung, die Vormauer sei erst im Jahr 447 hinzugefügt worden, muß revidiert werden. Die Vormauer wurde 477 zusammen mit anderen Mauerabschnitten lediglich gründlich repariert.

4. Das in der bisherigen Forschung kontrovers diskutierte Problem, ob das Goldene Tor bereits zur Zeit Theodosius' I. als ein Triumphtor erbaut worden war oder erst später, und zwar gleichzeitig mit der Landmauer von 
Theodosius' II. errichtet wurde, sollte zugunsten des Kaisers Theodosius II. entschieden werden, da zu vermuten ist, daß dieses Tor während der Errichtungszeit der Mauer (zwischen 405 und 413) in seiner heutigen Form noch nicht existierte. Es scheint, daß an seiner Stelle sich zunächst ein anderes Stadttor befand. Dieses war wahrscheinlich im Jahre 425 nach dem Sieg Theodosius' II. über den Usurpator Johannes Primicerius abgerissen und durch das existierende Monumentaltor ersetzt worden. Während der Neuerrichtung dieses Tors wurde auch die davor stehende Vormauer abgetragen und umgeleitet, was einen zusätzlichen Beleg für die Existenz der Vormauer vor dem Jahr 425 liefert.

5. Das Spolienensemble am Vortor des Goldenen Tors kann nicht länger mit Kaiser Ioannes V. Palaiologos bzw. Ioannes VI. Kantakuzenos in Verbindung gebracht werden. Das Vorwerk, das wahrscheinlich im früheren 9. Jahrhundert errichtet wurde, muß im 10. Jahrhundert und möglicherweise zur Zeit des Kaisers Nikephoros II. Phokas mit dem Rahmenwerk dekoriert worden sein. Dabei oder schon früher hat man die seitlichen Durchgänge aufgegeben.

6. Die in der wissenschaftlichen Literatur seit dem 19. Jahrhundert geläufige und bis heute verwendete Unterscheidung der Tore der Theodosianischen Landmauer in Militär- und Ziviltore ist nicht berechtigt, denn einige der bisher als Militärtore oder Nebentore bezeichneten Anlagen waren bedeutende Stadttore. Die in den Kurtinen angebrachten kleineren Öffnungen können hingegen als Nebentore bezeichnet werden. Während diese kleinen Öffnungen anscheinend mit profilierten Torgewänden dekoriert waren, hatten die Haupttore massive Laibungen und Torstürze, die bei späteren Verkleinerungen wieder eingesetzt worden sind.

7. Die fehlerhaften Identifikationen etlicher Tore haben die topographische Forschung vielfach auf falsche Fährten geführt. Die Tore sind folgendermaßen zu ordnen: Goldenes Tor $=$ Porta Aurea, Belgratkap $1=$ XylokerkosTor, Silivrikap $1=$ Pege-Tor, Tor zwischen den Tortürmen 39 und $40=$ Kalagros-Tor, Mevlevihanekapı = Rhesion- bzw. Myriandrion- oder Polyandrion-Tor, das Tor zwischen den Tortürmen 59 und $60=$ Romanos-Tor, Topkap $1=$ Pempton-Tor, Sulukulekap $1=$ Charisios-Tor, Edirnekap1 $=$ Johannes-Tor. Eine der wichtigsten Straßen der Stadt muß hinter Sulukulekapı begonnen und bis zur Markians-Säule geführt haben, während die Hauptstraße vom Romanos-Tor in Richtung Philadelphion verlief.

8. Es konnte nachgewiesen werden, daß das heutige Yedikulekapısı zwar eine osmanische Toranlage ist, doch befand sich ein byzantinisches Tor mit feinprofiliertem Gewände anscheinend wenige Meter weiter südlich und wurde im 18. Jahrhundert zugemauert, als man das osmanische Tor konzipierte. Weiterhin kann vermutet werden, daß das Tor zwischen den Tortürmen 39 und 40 (Kalagros-Tor) zur Errichtungszeit der Landmauer 
nicht als monumentale Anlage mit zwei Tortürmen konzipiert worden war, sondern hier eine einfache Nebenöffnung mit feinprofiliertem Gewände befand.

9. Die in der bisherigen Forschung kontrovers diskutierte Lage des Mesoteichion, wo sich im Jahre 1453 Kaiser Konstantin XI. Palaiologos Dragases und Sultan Mehmet II. gegenüber standen, ließ sind folgenderweise sichern: Der Mittelpunkt befand sich bei dem Tor zwischen den Tortürmen 59 und 60, also der als Romanos-Tor identifizierten Anlage. Die zugehörigen Abschnitte reichten nach Süden bis zum Mevlevihanekapı und nach Norden bis zum Lykos.

10. Mermerkule, das sich am Zusammenschluß der Theodosianischen Landmauer und der Marmara-Seemauer befindet, kann auf der Grundlage der Schriftquellen als das Polichnion des Kaisers Ioannes V. Palaiologos identifiziert und in die Zeit nicht lange vor 1389 datiert werden.

11. Die an die Theodosianische Landmauer bzw. an Tekfur Sarayı anschließende und bisher nicht sicher datierte Komnenen-Mauer muß im Jahre 1146/47 errichtet worden sein. Der südliche Teil der Mauer war möglicherweise auf Grund eines bisher noch nicht berücksichtigten Erdbebens im Jahre 1162 abgestürzt und wenig später in einem anderen Erscheinungsbild wiederhergestellt worden. Lediglich der nördliche Mauerabschnitt ab Kurtine 9/10 bis Turm 12, abgesehen von den späteren Reparaturen, gehörte der Ersterrichtungszeit an.

12. Die Komnenen-Mauer muß im Jahre 1285/86 durch Kaiser Andronikos II. Palaiologos repariert worden sein. Ein bisher unberücksichtigtes Erdbeben, das sich im Jahre 1315 ereignete, hat wahrscheinlich weitere Reparaturen an dieser Mauer verursacht und war im übrigen auch der bisher unbekannte Grund für wesentliche Reparaturmaßnahmen an der Hagia Sophia.

13. Bei der cloissonnéartigen Mauer zwischen Turm 13 und dem „Vorsprung“ an der Blachernen-Mauer wird es sich um die Begrenzung bzw. Substruktion eines bestimmten Trakts des Blachernen-Palastes gehandelt haben. Die Südwestecke dieser Begrenzung war gleichwohl der nördliche Endpunkt der Komnenen-Mauer und muß vor dieser errichtet worden sein. Turm 13 gehört seinem Mauerwerk zufolge wahrscheinlich in die frühpalaiologische Zeit.

14. Das in der Eroberungsgeschichte immer wieder erwähnte Palast-Tor ist mit dem Tor von Tekfur Sarayı zu identifizieren. Die bisher allgemein vertretene Meinung, daß die letzte Kaiserresidenz von Byzanz der BlachernenPalast gewesen sei, ist nicht zutreffend, da spätestens um 1344 Tekfur Sarayı als Kaiserpalast gedient haben muß. Das Kaligaria-Tor kann weiterhin mit dem heutigen Eğrikapı und das Gyrolimne-Tor nach wie vor mit dem Tor in Kurtine 11/12 der Komnenen-Mauer identifiziert werden. 


\section{Exkurs I}

\section{Die Hauptstraßen von Konstantinopel und ihre Beziehung zu den Toren der Landmauer}

Wie allgemein angenommen wird, verlief die Hauptstraße von Konstantinopel, die Mese, zwischen dem Milion und dem Kapitol bzw. Philadelphion. ${ }^{765}$ Diese Route entspricht etwa der heutige Divanyolu, Yeniçeriler Caddesi, Ordu Caddesi. ${ }^{766}$ Die Mese gabelte sich in mehrere Stränge, die zu den Toren der Theodosianischen Landmauer liefen. In der Forschung haben hauptsächlich drei Tore der Landmauer, und zwar - mit ihren heutigen türkischen Namen Edirnekapı, Belgratkapı und Altınkapı (Abb. 36) im Zusammenhang mit den zu ihnen führenden Hauptstraßen Beachtung gefunden, wobei sich hierfür feste Begriffe wie „Nordstang der Mese“ bzw. „Südstrang der Mese“ eingebürgert haben. ${ }^{767}$ Die übrigen Landmauer-Tore wurden unter dem Aspekt der zu ihnen führenden Straßen nur gelegentlich berücksichtigt (siehe Abschnitt 2.2.4.3.). Ausgehend von den Denkmälern bzw. Schriftquellen versuchte Schneider die innerstädtischen Straßen mit den Toren der Landmauer zu verbinden, wobei nur Sulukulekapı nicht einbezogen wurde. ${ }^{768}$ Thomov bemühte sich anhand von drei Buondelmonti-Ansichten von Konstantinopel das spätbyzantinische Straßensystem zu systematisieren. ${ }^{769}$ Er wählte dazu Beispiele aus, die ein recht einfaches Straßennetz aufweisen. Durch Vergleiche konnte er zeigen, daß innerhalb der von Straßen umgebenen Quartiere sich stets die gleichen Bauten befinden. Im folgenden Exkurs wird diese Methode zunächst auf solche Buondelmonti-Ansichten angewendet, die ein verzweigteres Straßensystem auf-

765 Siehe zuletzt Mango, Shoreline, 26; für die mögliche Breite der Mese, etwa $25 \mathrm{~m}$, ders., Développement, 27.

766 Auf dieser Strecke befinden sich u. a. Ayasofya, Çemberlitaş (Konstantin-Säule), Atik Ali Paşa Camii, Bayezıt Camii, Reste des Theodosius-Bogens vom Forum Tauri, Bayezıt Hamamı und Laleli Camii. Obwohl die Straßen moderne Gründungen sind, kennzeichnen die erwähnten Denkmäler noch ihren ursprünglichen Verlauf.

767 Berger, Regionen, 399, Anm. 260: „Als ,mittlere Straße’ kann nur der in der Mitte der Halbinsel liegende Straßenzug zwischen Milion und Kapitol bezeichnet werden, und tatsächlich läßt sich für die südliche Verlängerung zum Goldenen Tor und die erwähnte Straße nach Nordwesten der Name Mese den Quellen nicht eindeutig entnehmen. ... das Wort Mese wird allerdings auch allgemein für eine große Straße gebraucht und muß nicht immer die Hauptstraße von Konstantinopel meinen."

768 Schneider, Straßen, Plan 1 (Konstantinopel in spätbyzantinischer Zeit)

769 Thomov, New Information, 440-442, 445-447. 
weisen (Abb. 28). ${ }^{770}$ Das Straßennetz soll hier vor allem im Hinblick auf die Stadttore der Landmauer untersucht werden, zu denen nur Hauptstraßen hingeführt haben können.

Um die Wiedergabe des Straßennetzes auf seine Verläßlichkeit zu überprüfen, sollen zunächst die zu den Toren der Seemauern führenden Straßen betrachtet werden. An der Seemauer des Goldenen Horns sind in der Regel vier Tore mit Beischriften versehen: ${ }^{771}$ porta vlacherna, porta messis, porta piscaria, porta Judea. Das äußere rechte Tor ist durch die Beischrift $s$. dimitrius als Demetrios-Tor (Topkapı an der Seemauer) eindeutig zu identifizieren (Abb. 28). Darüber hinaus ist hier häufig die Demetrios-Kirche mit Beischrift dargestellt. Das unbezeichnete Tor zwischen Demetrios-Tor und porta Judea (Bahçekapı, Neorion-Tor) kann dann nur das Eugenios-Tor sein. ${ }^{72}$ Auf zahlreichen Buondelmonti-Ansichten ist das mittlere Tor der Mauer, auf das eine Hauptstraße zuführt, als porta messis beschriftet, ${ }^{773}$ womit das Tor am Zeugma, der byzantinische Vorläufer von Unkapanıkapı $(=\beta \alpha \sigma ı \lambda ı \kappa \eta ̀ ~ \pi u ́ \lambda \eta, ~ \pi o ́ \rho \tau \alpha ~ \pi \lambda \alpha-$ $\tau \dot{\varepsilon} \alpha$ ), gemeint ist. Da auf dieses Tor eine Hauptstraße rechts (unterhalb) des Pantokrator-Klosters (Beischrift: pantocratora) zuläuft, kann es sich auch hier nur um das mittlere Tor am Zeugma handeln. ${ }^{774}$ Demnach fehlen zwischen porta piscaria und mittlerem Tor das Perama-Tor (Zindankapı) und Odunkapı. Zwischen Blachernen-Tor und mittlerem Tor erkennt man noch zwei weitere Tore (das rechte ohne Straße), wobei das linke Ayakapı (= Theodosia-Tor) sein

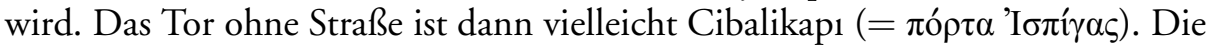
vom Eugenios-Tor, der porta Judea und der porta piscaria ausgehenden Straßen enden bei einer unbezeichneten Kirche. Da jedoch Zindankapı zu fehlen scheint, zu dem der Makros Embolos führte, ist der Straßenverlauf hier gestört. ${ }^{775}$

Die Tore an der Marmara-Seemauer lassen sich auf Grund der Straßenzüge und der benachbarten Gebäude ebenfalls identifizieren (von oben nach unten): Tor bei den Manganen (über der Straße oder darauf die Georgs-Kirche ${ }^{776}$ ), Ahırkapı (im Quartier darüber die Lazaros-Kirche), Tor am Bukoleon-Hafen,

770 Ich beziehe mich hier hauptsächlich auf folgende Buondelmonti-Ansicht: Venedig, Bibl. Marc., Ms. Lat. XIV.45 (= 4595), fol.123r: Barsanti, Buondelmonti, 184, Abb.61; Effenberger, Buondelmonti, Abb. 2.

771 Siehe die Beischriften bei Gerola, Vedute, 268-269; zu den einzelnen im folgenden erwähnten Toren und Bauten siehe zuletzt Effenberger, Buondelmonti, 23, 37-38, Tabellen I-III; vgl auch Schneider, Mauern und Tore, 105-107 und Berger, Ufergegend, $149-165$.

772 Zur Eugenios-Tor bzw. Eugenios-Turm siehe zuletzt Effenberger, Buondelmonti, 25. Auf Abb. 28 von Vfn. eingetragen.

773 In Abb. 28 von Verf. als „p.m“ eingetragen.

774 Auch bei Schneider, Straßen, Taf. I.

775 Bei Schneider zwischen Perama-Tor und Konstantins-Säule wiedergegeben.

776 Auf Abb. 28 von Verf. als „Tor“ eingetragen. 
vermutlich Çatladıkapı (darüber Kaiserpalast und Nea Ekklesia, darunter das als receptaculum papae bezeichnete Kloster im ehemaligen Hormisdas-Palast), ein Tor am Bukoleon-Hafen, zwei Tore zu beiden Seiten des Hafens Vlanga (portus volanga) - rechts das Juden-Tor (Yenikapı), links Davutpaşakapısı, dessen byzantinischer Name unbekannt ist. Das letzte Tor ist nur auf der BuondelmontiAnsicht in Venedig (Abb. 28) als porta enisia beschriftet und meint Narlıkapı. Auf der İstanbul-Karte von Matrakçı Nasuh aus dem Jahre 1537 werden an der Marmara-Seemauer sieben Tore ohne Namen angegeben. ${ }^{777}$ Auf der Berliner İstanbul-Ansicht von Piri Reis aus dem 18. Jahrhundert (Abb. 48) sind sieben Tore an der Marmara-Seemauer namentlich bezeichnet (siehe Exkurs II).

Im folgenden soll zunächst geprüft werden, ob das spätbyzantinische Straßensystem der Buondelmonti-Karten sich mit gesicherten Straßenzügen verbinden läßt. Dabei ist von folgender Hypothese auszugehen: Da die meisten Hauptstraßen beiderseits von Portiken eingefaßt waren, werden sie auch in spätbyzantinischer Zeit noch dem alten Straßenverlauf gefolgt sein, selbst wenn die Säulenhallen bereits zerstört waren. ${ }^{778}$ Die Mese und ihre Fortsetzungen sind auf fast allen Buondelmonti-Ansichten klar erkennbar. Auf der Ansicht in Venedig beginnt die Straße bei der Justinians-Säule bzw. zwischen Hagia Sophia und Hippodrom, also am Augusteion (Abb. 28, rot markiert). Nach einer gewissen Strecke gabelt sie sich in vier Stränge. Von dieser Hauptgabel verläuft der eine Strang vorbei an Theodosius- und Arkadius-Säule und überquert auch den Lykos. Links neben der Arkadius-Säule erscheint wie auf allen BuondelmontiAnsichten ein Torgebäude, durch das die Straße hindurchführt. Dieser Torbau ist zumeist als porta antiquissima pulchra beschriftet und wird in der Forschung zu Recht mit İsakapısı, dem ehemaligen Haupttor der konstantinischen Stadtmauer bzw. dem Tor am Exakionion identifiziert. ${ }^{779}$ Der Verlauf dieses Teilstücks der Mese entspricht etwa der heutigen Cerrahpaşa Caddesi. ${ }^{780}$ Man kann also vermuten, daß auch die übrigen Straßen, die zur Theodosianischen Landmauer führen, in ihrem Verlauf festgelegt waren. Wenig „westlich“ des Torbaus gabelt sich die Straße. Der eine Zweig führt südlich an der StudiosKirche vorbei zum theodosianischen Goldenen Tor, das auf mehreren anderen Buondelmonti-Ansichten als porta cresea beschriftet ist. Der andere Abzweig führt unter der Andreas-Kirche vorbei in einem großen Bogen zu einem wei-

777 Yurdaydın, Matrakçı, 8b.

778 Siehe dazu Mundell-Mango, Street, 29-51; Berger, Patria, 226-228, ders., Regionen, $391-392$.

779 Zur Lage des Tors und zum Exakionion siehe Van Millingen, Walls, 16-18; Mango, Triumphal Way 175-177; Effenberger, Buondelmonti, 35-36.

780 Vgl. Anm. 235. 
teren geöffneten Stadttor in der Landmauer. ${ }^{781}$ Um welches Tor es sich dabei handeln könnte, soll weiter unten untersucht werden.

Auf dem ersten Teilabschnitt zwischen Augusteion und Hauptgabel wird die Straße gesäumt von der Konstantins-Säule und von zwei Gebäuden im Quartier zwischen Straße und Hippodrom, die als domus constantini und sancto quaranta beschriftet sind. Das Haus des Konstantin wird mit der Euphemia-Kirche identifiziert und hat nichts mit dem Großen Palast zu tun. ${ }^{782}$ Die Lage der Vierzig-Märtyrer-Kirche läßt sich genau bestimmen. ${ }^{783}$ Demnach muß die Hauptgabel weiter westlich angenommen werden, was der üblichen Hypothese über deren Lokalisierung bei der Laleli Camii entsprechen dürfte, wobei jedoch die genaue Stelle unbekannt blieb. ${ }^{784}$ Hier kann nun auf eine osmanische Quelle hingewiesen werden, wonach das Philadelphion sich genau in der Nachbarschaft der Kızıltaş Mescidi (Moschee am Roten Stein) befunden haben muß. Nicht nur der Name der Moschee, sondern auch die von Ayvansarâyî Hüseyin Efendi gegebene Beschreibung bestätigen die Lage: ${ }^{785}$

Bu mescide Kızıltaş deyü tesmiyeye sebeb budur ki, mescidin binâsından mukaddem orada arka hammâlları istirahatleriçün bir seki taşı var imiş ki, kırmızı somaki imiş ... Mu'ahheren ol taşın ismi bu mescidi-i şerife lâkab olmuştur. Mahallesi vardır. Der Kurb-ı Lâleli Câmi’i

Der Grund, warum man diese Moschee als Kızıltaş Mescidi [Moschee am Roten Stein] bezeichnet, ist folgender: Vor dem Bau der Moschee soll sich dort ein Postament befunden haben, das aus rotem Porphyr bestand, worauf die Lastträger ausgeruht haben. Später wurde der Name dieses Steins auf diese verehrte Moschee übertragen. Sie hat ein eigenes Quartier. In der Nähe der Laleli Moschee

Es geht daraus hervor, daß es sich dabei nicht um einen hierhin verschleppten Stein, sondern um ein noch in situ befindliches Postament einer der Porphyrsäulen vom Philadelphion handelte, das möglicherweise Stufen hatte, auf die sich die Lastträger niedersetzen konnten. ${ }^{786}$ Die Mescid befand sich an der südöstlichen Seite der Laleli Camii, wo heute die Ordu Caddesi mit der Koska Caddesi zusammentrifft, und wo es auch im 16. Jahrhundert eine öffentliche

781 Einen ähnlichen Straßenverlauf hatte Schneider mit dem Xylokerkos-Tor (Belgratkapı) verbunden.

782 Effenberger, Buondelmonti, 35, Anm. 438.

783 Zisterne Nr. 24 bei Müller-Wiener, Topographie, Karte F 7; vgl. Berger, Patria, 317, 319-320. - Die in Abschnitt 2.3.2. erwähnte und nicht lokalisierte Vierzig-MärtyrerKirche, aus der Spolien für das Polichnion des Kaisers Ioannes V. Palaiologos entnommen worden waren, hat mit dieser Kirche nichts zu tun.

784 Zusammenstellung der verschiedenen Hypothesen bei Bauer, Denkmal, 228-233. Mango, Développement, 76; siehe zuletzt Denis Feissel, Le Philadelphion de Constantinople: inscriptions et écrits patriographiques, in: Comptes Rendus de l'Académie des Inscriptions et Belles-Lettres 2003, 495-523, bes. 515, 521.

785 Hadîkat ül Cevâmî, 217.

786 Nach Speck, Konzept, 147, handelte es sich dabei um Halbsäulen! 
Straße gab, die Nachfolgerin der Mese und Vorläuferin der heutige Ordu Caddesi gewesen sein muß. ${ }^{787}$ Das Postament wird dicht an dieser öffentlichen Straße gestanden haben. Demnach kann das Philadelphion auf der Kreuzung der heutigen Ordu Caddesi, Koska Caddesi und Fethi Bey Caddesi lokalisiert werden, die noch die in Richtung Goldenes Horn bzw. zum Marmarameer führenden Straßen widerspiegeln. ${ }^{788}$ Etwas $150 \mathrm{~m}$ südwestlich dieser Stelle befindet sich die Bodrum Camii (Myrelaion-Kirche), wo das anpassende Fußfragment einer der beiden 1204 nach Venedig verschleppten Tetrarchengruppen vom Philadelphion gefunden wurde. Mit dem Postament aus Porphyr wäre somit der Standort einer der beiden von Verzone rekonstruierten Porphyrsäulen wiedergewonnen. ${ }^{789}$

Der in Richtung Landmauer verlaufende sog. Nordstrang (Abb. 28, rot markiert) führt auf den Buondelmonti-Ansichten von der Hauptgabel unterhalb der Apostel-Kirche und der als s. johannes de petra beschrifteten Kirche vorbei zu einem aus drei Tangenten gebildeten Straßenknoten, der wiederum mit einem geöffneten Tor - dem letzten vor Tekfur Sarayı - in Verbindung steht. ${ }^{790}$ Wie bereits erwähnt, ist dieses Tor auf einigen Buondelmonti-Veduten als porta sancti Ioannis bezeichnet (siehe Abschnitt 2.2.4.3.) und muß mit Edirnekapı identifiziert werden. Auf den Darstellungen laufen an der Stadtseite des Tors zwei Straßen zusammen: Eine vom Blachernen-Tor heranführende Hauptstraße (die heutige Kesmekaya Caddesi scheint noch teilweise ihrem Verlauf zu folgen) sowie eine Straße, die das Quartier mit der als s. martha beschrifteten Kirche kreisförmig umrundet und sich mit einer anderen Straße verbindet.

Byzantinische Schriftquellen und russische Pilger erwähnen ein Kloster der Kyra Martha. ${ }^{791}$ Die Gründerin Kyra Martha war die älteste Schwester Kaiser Michaels VIII. Palaiologos, Maria Palaiologina. ${ }^{792}$ Als Gründungsdatum wird die Zeit zwischen 1261 und 1266 angenommen. ${ }^{793}$ Schneider identifizierte die Kirche mit der Sekbanbaşı Mescidi, ${ }^{794}$ Eyice brachte sie - ausgehend von den Buondelmonti-Veduten - mit der Manastır Mescidi in Verbindung. ${ }^{795}$ Laurent plazierte sie nach den Berichten der russischen Pilger in der Nähe des Kon-

787 Tahrir Defteri I, 383, Nr. 2252. - Der Ort der Moschee ist noch eingetragen bei MüllerWiener, Topographie, Plan E 7/6.

788 Siehe Kent Atlası, 286, C 5/6; vgl. Ayverdi, İstanbul Haritası, C.3, wo die Straßennamen von den heutigen abweichten. Auf diesem Plan heißt die heutige Koska Caddesi Camii Sokak und die heutige Ordu Caddesi Koska Caddesi.

789 Verzone, Philadelphion, 8-14; Naumann, Rundbau, 209.

790 Siehe Anm. 448.

$791 \mathrm{Zu}$ den Quellen siehe Janin, Eglises, 324-325; Kidonopoulos, Bauten, 51.

792 Siehe Kidonopoulos, Bauten 51-52; Effenberger, Bebaia Elpis, 256.

793 Kidonopoulos, Bauten, 52.

794 Schneider, Byzanz, 61.

795 Eyice, Kyra Martha, 139-168. 
stantinos-Lips-Klosters, was in der jüngeren Sekundärliteratur allgemeine Zustimmung gefunden hat. ${ }^{796}$ Diese Lage steht aber im Widerspruch zu der Aussage der Buondelmonti-Kopien. ${ }^{797}$ Die Kyra-Martha-Kirche befindet sich hier nahe einem Tor der Landmauer und wird von einem Straßenbogen umrundet. Auf seinem Plan hat Schneider einen ähnlichen „konvergierenden“ Straßenverlauf hinter Topkapı angenommen. ${ }^{798}$ Auf der Karte von Kauffer/ Lechevalier von 1786 geht von diesem Tor noch eine gerade Straße aus, die bei der Molla Gürani Camii endet. Sie könnte eine frühosmanische Straße widerspiegeln, da nämlich das Romanos-Tor damals zugemauert war und wahrscheinlich die damit verbundene Straße nicht mehr benutzt wurde. Diese Straße scheint aber im 19. Jahrhundert aufgegeben worden zu sein, da zwischen Romanos-Tor und Topkapı eine Tramstraße erbaut wurde, die mit dem Straßenbogen um die Martha-Kirche auf den Buondelmonti-Karten identisch sein könnte. ${ }^{799}$ Innerhalb dieses Bogens an der Südseite des Topkapı befindet sich die Bayezıt Ağa Camii. Sie stammt ursprünglich aus dem 15./16. Jahrhundert, wurde aber Anfang des 20. Jahrhunderts fast gänzlich erneuert. ${ }^{800}$

Ein Kyra-Martha-Quartier (Kiramarte Mahallesi) wird noch in einer in arabischer Sprache verfaßten Stiftungsurkunde Mehmets II. aus dem Jahr 1472 erwähnt, doch ist die Lokalisierung nicht sicher. ${ }^{801}$ Es ist möglich, daß das Kloster - obwohl es unmittelbar nach der Eroberung zerstört worden sein soll ${ }^{802}$ - noch längere Zeit aufrecht stand. ${ }^{803}$ Die Bayezıt Ağa Camii, deren Stiftungsurkunde aus dem Jahre 1520 stammt, ${ }^{804}$ könnte theoretisch Nachfolgerin der Kirche der Kyra Martha sein.

Damit können wir zum sog. Nordstrang der Mese zurückkehren: Der Nordstrang führt also nicht geradewegs zu Edirnekapı, wie man annehmen möchte, sondern zweigte einmal in Richtung Blachernen-Tor $a b$, und zum anderen mündet er in den Straßenbogen, der um die Kyra Martha-Kirche zu der darunterliegenden Straße führt. Da alle relevanten Buondelmonti-Ansichten diese besondere Situation vor dem Johannes-Tor genau so wiedergeben, kann geschlossen werden, daß der sog. Nordstrang - zumindest in spätbyzantinischer

796 Laurent, Kyra Martha, 320; vgl. Janin, Eglises, 324-326; Majeska, Travelers, 33, Effenberger, Bebaia Elpis, 256.

797 Eyice, Kyra Martha, 139; vgl. Effenberger, Buondelmonti, 50.

798 Schneider, Straßen, Plan I.

799 Ayverdi, İstanbul Haritası, E5.

800 Ayverdi, Fatih Devri III, 323-324.

801 Ergin, Vakfiye, 42/9; Ayverdi, Mahalleler, 33.

802 Kidonopoulos, Bauten, 51. - Da die osmanischen Quellen hinsichtlich der byzantinischen Denkmäler noch nicht ausreichend bearbeitet worden sind, sollte man $\mathrm{m}$. E. von solchen vorschnellen Behauptungen Abstand nehmen.

803 Die Quartiere entwickelten sich in der Regel um eine Moschee; siehe Schneider, Quartiere, 151.

804 Tahrir Defteri I, 395, Nr. 2316. 
Zeit - bereits ein Stück vor Edirnekapı endete und sich hier mit anderen Straßen verband.

Von der Hauptgabel oder wenig versetzt zweigt eine weitere Straße ab (Abb. 28, grün markiert), die allerdings „nördlich“ an der Theodosius-Säule vorbeiführt, weil diese zumeist falsch eingetragen ist. Da sie den Lykos überquert, muß sie entlang der Nordostflanke des Xerolophos und südlich des Lykos bergauf zur Landmauer verlaufen sein. Sie endet entweder bei einem Tor oder läuft stumpf gegen die Innenseite eines Turms. Da auf allen BuondelmontiKopien der Lykos erst innerhalb der Stadt aus drei Quellen entspringt, bleibt im Bereich der Mauer leider unklar, welche Tore nördlich und welche südlich des Bachs angenommen werden müßten. Das zuletzt erwähnte Tor wird auf einigen Buondelmonti-Ansichten als porta camidi oder chamidi bezeichnet. ${ }^{805}$ Barsanti hat aber darauf hingewiesen, daß dies eine Ableitung von Kosmidion ist. ${ }^{806}$ Auf einer Buondelmonti-Ansicht in Venedig befindet sich an dieser Stelle die Bezeichnung porta pigti ${ }^{807}$ womit eindeutig das Pege-Tor (Silivrikapı) bezeichnet ist, was vom Verlauf des Abzweigs einer Straße südlich des Lykos genau hinkäme. Das Tor war also zur Entstehungszeit von Buondelmontis Karte passierbar. Nur auf einer Buondelmonti-Ansicht in Paris ist im mittleren Bereich der Landmauer noch ein als sanctus Romanus bezeichnetes Tor mit Brücke angegeben (Abb. 46), obwohl das Tor zur Entstehungszeit des Bildes (nach 1457/58) bereits nicht mehr benutzbar war. Da die Buondelmonti-Ansichten an der Landmauer nur drei passierbare Tore verzeichnen, müssen die übrigen unzugänglich, d.h. noch (seit 1389/90?) vermauert gewesen sein. Zwischen Goldenem Tor und Pege-Tor betrifft das Belgratkap1, und zwischen Pege-Tor und Johannes-Tor (Edirnekapı) alle Tore: Rhesion-Tor, Romanos-Tor, Pempton (Topkap1) und Charisios-Tor (Sulukulekap1, Abb. 36). Die mittlere Straße, die auf der Kopie in Venedig nördlich des Pege-Tors, aber südlich des Lykos vor dem Turm der Mauer endet, muß demnach ursprünglich zu einem der beiden zwischen Pege-Tor und Lykos gelegenen Tore geführt haben. In Frage kämen hierfür nur Rhesion-Tor und Romanos-Tor.

Diese mittlere Straße war also eine wichtige Hauptstraße. Sie begann wohl wenig westlich der Hauptgabel bzw. war die Verlängerung der Straße, die sich zwischen Milion und Philadelphion erstreckte, wo der Abzweig des sog. Nordstrangs der Mese meist angenommen wird, verlief westlich und kann ei-

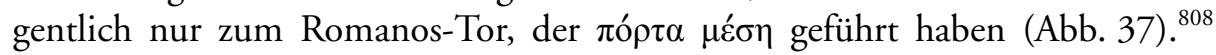
Nicht nur der Name des Tors erinnert an die Hauptstraße von Konstantinopel,

805 Barsanti, Buondelmonti, 192, Abb.72; 199, Abb. 76; 206, Abb. 80.

806 Barsanti, Buondelmonti, 200.

807 Barsanti, Buondelmonti, 202, Abb. 77.

808 Schneider, Straßen, Taf. 1, verband dieses Tor (ohne Namen) mit einer vom Bus ausgehenden Straße. 
sondern auch ein Bericht aus dem Jahre 1453 weist indirekt darauf hin, wie die Straße verlaufen sein kann. Barbaro teilt mit: ${ }^{809}$

Vignando i Turchi furioxamente verso la piaza, che sono mia cinque luntan, dove che i fexe l'intrada che fo de san Romano, zonti che i fo a la piazza, subito queli de lor Turchi si montò suxo una tore dove che iera levado san Marco

Die Türken begaben sich in rasender Eile zu dem Platz, der fünf Meilen vom Romanos-Tor entfernt liegt, wo sie eingedrungen waren. Kaum waren sie auf dem Platz angelangt, stiegen einige Türken sofort auf den Turm, der beim heiligen Marcus gelegen war

Die Kirche des heiligen Marcus wird nach den Quellen im allgemeinen in der Nachbarschaft des Tauros angenommen, ${ }^{810}$ der etwa am Ort der heutigen Philosophischen Fakultät der Universität İstanbul lokalisiert werden kann, also auf der Ordu Caddesi, d. h östlich vom Philadelphion auf der Mese (Abb. 37). Aus Barbaro geht hervor, daß zwischen Romanos-Tor und Philadelphion 1453 eine direkte Straße verlief. Es ist daher die Frage zu stellen, ob die Byzantiner unter "Mese“ nur die Strecke zwischen Milion und Philadelphion verstanden haben, oder ob damit nicht vielmehr die gesamte Route bis zum Romanos-Tor gemeint gewesen sein könnte. Wenn Belgratkapı, Mevlevihanekapı, RomanosTor, Sulukulekapı und vielleicht auch Topkapı zur Zeit Buondelmontis schon nicht mehr passierbar waren, dann verwundert es nicht, daß die ursprünglich zu diesen Toren führenden Hauptstraßen - mit Ausnahme der mittleren Straße auf den Buondelmonti-Ansichten nicht mehr dargestellt sind, was nicht bedeutet, daß sie nicht mehr existierten.

In Abschnitt 2.4.3. wurde bereits vermutet, daß die Markians-Säule (Abb. 212) an einer bedeutenden Hauptstraße gelegen haben muß. Diese Straße führte, wie oben diskutiert, zu Sulukulekapı, dem einstigen Charisios-Tor (Abb. 37). Der dreieckige Stadtgrundriß und der wie mit dem Zirkel geschlagene Bogen der Theodosianischen Landmauer legen es nahe, hier von einem System von Radialstraßen auszugehen, die im Stadtinneren von der eigentlichen Mese abzweigten. Auf seinem Plan läßt Bauer die Straße von Edirnekap1 („Charisios-Tor“) zunächst parallel zur Südwestseite der Aetios-Zisterne und der Apostel-Kirche verlaufen, postuliert dann aber einen Knick in Richtung Markians-Säule, da deren Sockel mit seiner Schauseite in Richtung Fevzi Paşa Caddesi orientiert ist, und weiter vorbei an der Polyeuktos-Kirche zum Phil-

809 Pertusi, La Caduta I, 34/809-815.

810 Janin, Eglises, 327; Berger, Patria, 501; Berger, Tauros, 24. - Pertusi, La Caduta, 363, Anm. 148, nimmt diesen Maß als „miglia“ an hat diese in $7,5 \mathrm{~km}$ umgerechnet, weswegen er die Kirche nahe zum Augusteion plazieren mußte. Die Entfernung zwischen Romanos-Tor und dem erwähnten Turm - rund 3,4 km - ergäben für eine „Meile“ $680 \mathrm{~m}$, also 1000 einfache Schritte. 
adelphion. ${ }^{811}$ Dabei bezieht er für den letzten Abschnitt auch die nachgewiesenen Terrassierungen in seine Überlegungen ein. Allerdings trifft es nicht zu, daß Markians-Säule und Atrium der Polyeuktos-Kirche ,in einer Linie parallel zum Valens-Aquädukt liegen." Nimmt man vor der Nordseite der PolyeuktosKirche gemäß ihrer Flucht eine Straße an, würde diese in den anzunehmenden Platz einmünden, auf dem die Markians-Säule steht Abb. 37). Die vom Charisios-Tor (Sulukulekapı) in die Stadt führende Straße kann durchaus einen Abzweig zum Deuteron gehabt haben, schon weil der Weg Justinians I. vom Charisios-Tor über das Deuteron zur Apostel-Kirche dies nahelegt (siehe Abschnitt 2.2.4.3.), doch wird ihr Hauptstrang über den Platz mit der MarkiansSäule verlaufen sein. Die Tatsache, daß sich für diese Straße in den Quellen bislang kein Prozessionsweg nachweisen läßt, spricht nicht gegen ihre Existenz, ebensowenig, daß sie auf den Buondelmonti-Ansichten nicht mehr eingetragen ist. Die von Edirnekapı in die Stadt führende Straße kann nur über die heutige Şehzadebaşı Caddesi weiter nach Osten verlaufen sein, wo Anfang des 20. Jahrhunderts noch die Reste einer Portikusanlage beobachtet wurden, und endete am Tauros (Abb. 37). ${ }^{812}$

Man muß also sämtlichen zu den Haupt- und Nebentoren führenden Straßen künftig mehr Aufmerksamkeit schenken und auch die möglicherweise dort angrenzenden Gebäude in die Überlegungen einbeziehen. ${ }^{813}$

811 Bauer, Denkmal, 230; ebd., 166, Abb. 47.

812 Schneider, Straßen, 72.

813 Gemeinsam mit Arne Effenberger bereite ich eine Untersuchung über die Konstantinsmauer und das vortheodosianische Straßensystem vor. 


\title{
Exkurs II
}

\section{Zur Vorlage der İstanbul-Karten im Berliner und Londoner Exemplar des Kitâb-1 Bahriye von Piri Reis ${ }^{814}$}

\author{
Das Berliner Exemplar des Kitâb-1 Bahriye ${ }^{815}$ (Buch der Seefahrt) von Piri Reis \\ war noch im 18. Jahrhundert im Besitz der Haremsdamen und wurde im Jahre \\ 1789 durch den Preußischen Botschafter Friedrich Heinrich von Diez von \\ einem im Topkapı Palast tätigen Eunuchen erworben. Es befindet sich in der \\ Orientabteilung der Staatsbibliothek zu Berlin unter der Signatur Diez A
}

814 Eine der hervorragendsten Persönlichkeiten für die Seefahrtsgeschichte des 15./16. Jahrhunderts ist der an der Marmaraküste der Türkei in Gelibolu um 1465/70 geborene osmanische Kapitän Muhittin Piri, geläufiger als Piri Reis (Kapitän Piri). Er ist nicht nur als ein Seemann, sondern auch als einer der prominentesten Kartographen seiner Zeit bekannt, u.a. stammt die älteste bekannte Karte von Amerika (Columbus-Karte von 1498) aus seinem Feder. Über sein Leben erfährt man aus seinem Kitâb-1 Bahriye. Bereits im Kindesalter begann er in der Flotte seines Onkels Kemal Reis, der ein berühmter Pirat war, im Mittelmeer zu segeln. Als Bayezit II. (1481-1512) den inzwischen zur Legende gewordene Kemal Reis für seine Marine gewann, stand auch der Neffe Piri ab 1495 dem Sultan zu Diensten, vor allem während des Kriegs gegen Venedig. Auch nach dem Tod seines Onkels (1511) und der Thronübernahme durch Sultan Selims I. (1512) setzte Piri Reis seine Tätigkeit für die osmanische Marine fort. Innerhalb dieser Zeit entstanden mehrere Karten, so von Kairo und vom Nil sowie die dem Sultan geschenkte Karte von Amerika (1513). Zum Leben von Piri Reis siehe İnan, Piri Reis; zur Amerika-Karte siehe Kahle, Columbus Karte; zu Kemal Reis siehe Tavârih-i Âli Osman VIII, 145-146. Für eine venezianische Darstellung von Kemal Reis während einer Seeschlacht zwischen Venezianern und Türken siehe Müller-Wiener, Häfen, Taf. 58, Abb. 2.

815 Bereits vor 1521 begann Piri Reis mit der Arbeit an seinem berühmten Portulan Kitâb-1 Bahriye über die Örtlichkeiten des Mittelmeers und des Ägäischen Meers, in der Absicht, ihn Sultan Selim I. zu schenken (Kurzversion). Eine zweite und unfangreichere Version (Langversion) des Buches wurde 1525/26 Sultan Süleyman I. (1520-1573) überreicht. Im Laufe der Zeit entstanden Kopien beider Versionen, die heute in zahlreichen Museen bzw. Bibliotheken aufbewahrt werden. Die Kopien sind in einigen Fällen durch Kolophone datiert, die hin und wieder Hinweise auf die jeweiligen Besitzer geben, u. a. Seeleute oder Staatsmänner. Die früheste datierte Kopie der Langversion befindet sich in der Bibliothek des Topkapı Sarayı (Ms. TSMK. H. 642). Sie besitzt kein Kolophon. Es wird angenommen, daß es sich dabei um das Sultan Süleyman geschenkte Exemplar handelt. Die früheste Kopie der Kurzversion von 1554 wird in der Sächsischen Landesbibliothek zu Dresden aufbewahrt (Ms. Eb. 389). Nicht alle Abschriften enthalten die Karte von İstanbul. Für eine umfangreiche Diskussion über beide Versionen siehe Orbay, Ottoman Maps, 123-133. 
fol. 57. ${ }^{816}$ Die darin enthaltene İstanbul-Karte wurde erstmals von Oberhummer veröffentlicht. ${ }^{817}$ Die Stadt ist auf zwei Folios wiedergegeben (28a/b). Sie zeigt die Altstadt (ehem. Konstantinopel), die Vororte (teilweise) westlich der Theodosianischen Landmauer, Pera (Galata und Kasımpaşa), die asiatische Seite (Üsküdar, Kadıköy, Moda, Fenerbahçe) und die Prinzeninseln. Prominente Örtlichkeiten sowie die wichtigsten Denkmäler tragen jeweils eine Beischrift. An der Seite des Goldenen Horns wurden die Tornamen von einer zweiten Hand in etwas dunklerer Schrift wiederholt (Abb. 48).

Obwohl die Karte oft in die kunsthistorische bzw. topographische Forschung einbezogen wurde, ist niemals die Frage gestellt worden, inwieweit sie noch mit der Urkarte von Piri Reis übereinstimmen könnte, von der wir ohnehin nur Kopien besitzen. Im Rahmen ihrer Dissertation beschäftigte sich jüngst İffet Orbay auch mit der Berliner Karte und behandelte mehrere der darauf dargestellten Denkmäler. ${ }^{818}$ Für ihre Entstehungszeit akzeptierte sie den bereits von Oberhummer vorgeschlagenen terminus post quem 1663, da auf der Karte die in diesem Jahr vollendete Yeni Valide Camii im Stadtteil Eminönü erscheint. Die Autorin versuchte weiterhin Hinweise für einen terminus ante quem zu gewinnen, wobei sie auf drei Punkte aufmerksam machte: Erstens die Darstellung eines nach dem Jahre 1722 nicht mehr existierenden İmaret an der asiatischen Seite, zweitens das Vorkommen der Arkadius-Säule, die 1715 abgetragen worden sei, drittens das Fehlen der 1707 erbauten Çorlulu Ali Paşa Moschee im Tersane-Gebiet von Kasımpaşa. ${ }^{819}$ Orbay neigt der Zeitspanne nach 1663 und vor $1707 \mathrm{zu}$, da der Kopist, der das Tersane von Kasımpaşa so detailliert dargestellt hatte, nicht die Çorlulu Ali Paşa Moschee vergessen haben könne. Sie wies weiterhin auf die Ähnlichkeit zwischen der Berliner Karte und der Kopie in der Khalili Collection in London hin (im folgenden Londoner Karte) (Abb. 47) ${ }^{820}$ und nahm an, daß eine weitere Version, die sich in der Pariser Nationalbibliothek befindet und um 1651 datiert wird (im folgenden

816 Piri Reis, Kitâb-1 Bahriye, Berlin, Staatsbibliothek, Orientabteilung, Sign. Diez A fol.57. - Siehe W. Pertsch, Die Handschriften-Verzeichnisse der Königlichen Bibliothek zu Berlin, Bd.6: Verzeichnis der Türkischen Handschriften, Berlin 1889, 203-210, Nr. 184, Blatt XXVIII, $1-2$.

817 Oberhummer, Konstantinopel, 22-23, Taf. XXII. - Eine Kopie der Karte mit zusätzlichen Beischriften scheint bereits vor dem Jahr 1900 durch Ahmet Muhtar Paşa in seinem handschriftlich verfaßten Buch Konstantiniye (226) benutzt worden zu sein. Die Handschrift befindet sich in der Bibliothek der Universität İstanbul unter der Signatur TY 5010. Die Karte wurde mit dieser Referenz von Bostan veröffentlicht siehe Bostan, Bahriye Teşkilâtı, Taf II, obere Abbildung.

818 Orbay, Ottoman Maps, 117-290.

819 Orbay, Ottoman Maps, 287, Anm. 392.

820 London, Khalili Collection, MSS 718; siehe dazu Soucek, Mapmaking, 132-161; Rogers, Empire of the Sultans, 124-125. 
Pariser-Karte), als Vorlage für die Berliner und Londoner Karte gedient haben müsse. ${ }^{821}$

Während in einigen früheren Kopien des Kitâb-1 Bahriye İstanbul von der asiatischen Seite aus betrachtet wird ${ }^{822}$ und an die Karte von Vavassore ${ }^{823}$ oder Sadeler ${ }^{824}$ erinnern, bilden spätere Kopien - einschließlich der Pariser, Berliner und Londoner - die Stadt von der Pera-Seite, also von Norden her ab. In allen drei Ansichten ist die Stadt auf zwei Folios verteilt, bei fast identischen Umrissen und ähnlich dichter Füllung mit Häusern und Monumenten. Trotzdem ist es fraglich, ob die Pariser Fassung als Vorlage für die Londoner und Berliner Karte gedient haben kann, da die beiden letztgenannten Gemeinsamkeiten enthalten, die weder auf der Pariser noch auf sonst einer Piri Reis-Kopie oder einer Darstellung von İstanbul vorkommen. Diese für die Topographie İstanbuls wichtigen Punkte sollen im folgenden diskutiert werden.

Sowohl auf der Berliner als auch auf der Londoner Karte wurde die Südwestecke der Stadt zwischen Yedikule und Mermerkule in einer Weise dargestellt, die dem Betrachter sofort ins Auge fällt: Vom südlichsten Turm von Yedikule geht eine mit Türmen bewehrte Mauer aus und trifft im Süden auf einen Turm der Marmara-Seemauer. Dadurch entsteht an der Südwestecke der Stadt - in der Nachbarschaft von Yedikule - ein von Mauern umgebenes Areal, das auf der Londoner Karte als Bucakbağı (Eckgarten) beschriftet ist. ${ }^{825}$ Berger sah es - ausgehend von der Berliner Karte - als „merkwürdiges Detail“ an und mahnte hinsichtlich der Deutung zur Vorsicht. ${ }^{826}$ Orbay ging kurz darauf ein und behandelte das Areal unter den Gartenanlagen İstanbuls im 17. Jahrhundert. ${ }^{827}$ Wie osmanische Schriftquellen bestätigen, handelt es sich dabei um eine Gartenanlage mit dazugehöriger Mahalle, die bereits in der ersten Hälfte des 16. Jahrhunderts vorhanden waren. ${ }^{828}$ Es stellt sich aber die Frage, ob diese Gar-

821 Paris, Bibliothèque Nationale de France, Ms. supp. Turc 956, fol.434v. - Farbaufnahme bei Du Thanney, Matrakçı, 89, Abb. 64.

822 Für einen Vergleich siehe die Abbildungen bei Orbay, Ottoman Maps, 469-507.

$823 \mathrm{Du}$ Tanney, Matrakçı, 103, Abb. 73.

824 Du Tanney, Matrakçı, 105, Abb. 74.

825 Um einem möglichen Irrtum vorzubeugen, soll hier vermerkt werden, daß eine in Genf im Rahmen einer Ausstellung verkaufte Postkarte, worauf nur diese Ecke der Londoner Karte wiedergegeben ist, bei Ahunbay, Yedikule, 199, Abb.6, mit der Referenz Musées d'art et d'histoire, Geneva, abgebildet ist. Soweit mir bekannt, befindet in diesem Museum keine Kopie des Kitâb-1 Bahriye von Piri Reis.

826 Berger, Langa Bostanı, 477, Anm. 37.

827 Orbay, Ottoman Maps, 271.

828 Orbay, Ottoman Maps, 269-270, machte darauf aufmerksam, daß im 17. Jahrhundert der Name Bucakbağ durch Evliya Çelebi, 237, 300, erwähnt wird. In einer nach der Arbeit von Orbay veröffentlichten früheren osmanischen Quelle kommt der Garten im Zusammenhang mit einigen Stiftungsurkunden vor, so einer aus dem Monat Zilkâde 955 (Dezember 1548); siehe Tahrir Defteri II, 647-648. 
tenanlage tatsächlich durch eine Quermauer gesperrt war. Dieser Zustand ist uns nämlich aus keiner schriftlichen oder bildlichen Quelle aus dem 17. Jahrhundert bekannt ist.

Auf beiden Karten bildet Mermerkule die südwestlichste Ecke des Areals. Wenn es sich dabei, wie oben dargelegt, um das Polichnion von Ioannes V. Palaiologos handelte, kann ein Kopist des 17. Jahrhunderts höchstens noch Reste der Mauer bzw. Türme gesehen haben, da unseres Wissens die Mauer wenn auch nur teilweise - bereits in byzantinischer Zeit zerstört worden war (siehe Abschnitt 2.3.2.). Der Kopist der gemeinsamen Vorlage der Berliner und Londoner Karte hat entweder aus eigener Phantasie die Mauerreste vervollständigt oder die alte byzantinische Umfassungsmauer war in diesem Bereich irgendwann wieder instand gesetzt worden. Es ist auch denkbar, daß die vollständige Ummauerung auf einer älteren Karte, die vom Kopisten der gemeinsamen Vorlage benutzt wurde, noch wiedergegeben war. Es würde bedeuten, daß der Kopist zwar nicht die Mauer, wohl aber diese ältere Karte kannte, was plausibeler erscheint.

Das läßt sich vielleicht mit folgenden Beobachtungen beweisen: Auf der Londoner Kopie ist an der östlichen Seite der als Davutpaşakapısı beschrifteten Toranlage an der Marmara-Seemauer ein herausragender und mit der Mauer verbundener Eckturm sowie ein im Meer freistehender Turm angebracht und als „Kulle-i Hamza“ beschriftet. Es handelt sich dabei um das in byzantinischer Zeit „Belisarios-Turm “829 und in den osmanischen Quellen „Papaz-Kulesi“830 genannte Bauwerk. Um den Turmsockel wurden einige Felsbrocken angedeutet, die auf die einst hier befindliche Mole hinweisen. Eine ähnliche Darstellung, jedoch ohne Felsbrocken und Beischrift, begegnet auch auf der Berliner Karte, wobei der Eckturm detaillierter wiedergegeben ist. Es handelt sich hier eindeutig um den byzantinischen Theodosius-Hafen, der in spätbyzantinischer Zeit durch zwei Türme verstärkt wurde. ${ }^{831}$ Orbay zufolge handle es sich dabei um eine Neuheit in den Piri Reis-Karten, doch ging sie nicht darauf ein, weshalb diese hier plötzlich auftritt und woher der Kopist die Anregung dazu erhalten haben könnte. ${ }^{832}$ Der Theodosius-Hafen sah nämlich im 17. Jahrhundert anders aus, als er auf diesen beiden Karte dargestellt wurde. Der Hafen war, wie die Düsseldorfer Kopie einer Buondelmonti-Ansicht eindeutig zeigt, bereits vor 1490 zugeschüttet worden. ${ }^{833}$ Nur der östliche Teil war, Petrus Gyllius zufolge, um 1550 noch benutzbar. ${ }^{834} 1760$ wurde auch dieser Teil mit dem Bauaushub der

829 Effenberger, Buondelmonti, 31, Nr. 13.

830 Siehe als Beispiel Eremya Çelebi Kömürcüyan, 3, 78.

831 Effenberger, Buondelmonti, 31-33, Nr. 13.

832 Orbay, Ottoman Maps, 270.

833 Effenberger, Buondelmonti, 31-33, Nr. 13.

834 Gyllius, Topographia, IV, 8, 212-213. 
Laleli Camii zugeschüttet, und es entstand die Yenimahalle. ${ }^{835}$ Der offene $\mathrm{Zu}$ stand des Hafens kann also keinem Kopisten des 17. Jahrhunderts mehr bekannt gewesen sein, es sei denn, er hat eine Vorlage aus dem 15. Jahrhundert, womöglich eine vor 1490 angefertigte, verwendet. ${ }^{836}$

Diese Annahme läßt sich durch eine weitere Beobachtung stützen: Sowohl auf der Londoner (Abb. 47) als auch auf der Berliner Karte (Abb. 48) wurde die Theodosianische Landmauer entsprechend ihrer tatsächlichen Gestalt als Doppelmauer mit Graben wiedergegeben. In der Anzahl der Tore weichen beide Kopien jedoch von einander ab - ein Detail, dem bisher keine Aufmerksamkeit geschenkt wurde. Die Londoner Karte stellt von Süden nach Norden die folgenden fünf Tore jeweils mit einer Beischrift dar: Bâb-1 Yedikule, Bâb-1 Silivri, Bâb-1 Yeni (Mevlevihanekapı), Bâb-1 Top und Bâb-1 Edirne. Doch wurde nördlich der als Bâb-1 Edirne beschrifteten Toranlage ein weiteres Tor eingefügt, das auf der gleichen Flucht wie die Fatih Camii steht. Dieses Tor besitzt jedoch keine Beischrift. Darüber hinaus wurde die Mihrimah Camii, die auf der gleichen Ebene mit der die Fatih Camii, also bei diesem Tor stehen müßte, nicht dort, sondern hinter dem südlich benachbarten Tor plaziert, das irrtümlich als Edirnekapı beschriftet wurde. Diese Unstimmigkeit bedarf einer Erklärung. Den osmanischen Quellen zufolge waren im 17. Jahrhundert an der Landmauer nur fünf Tore passierbar, und zwar Yedikulekapısı unmittelbar nördlich des Goldenen Tors (Altınkapı), Silivrikapı, Yenikapı (= Mevlevihanekapı), Topkapı und Edirnekapı. Belgratkapı zwischen Yedikule und Silivrikapı, das RomanosTor und Sulukulekapı zwischen Topkapı und Edirnekapı sowie weitere kleinere Nebenöffnungen waren zugemauert (Abb. 36). Auch die im Jahre 1537 gezeichnete Karte von Matrakçı Nasuh zeigt nur fünf Tore an der Landmauer analog zu späteren Aussagen. ${ }^{837}$ Sulukulekapı war noch um 1900 geschlossen, ${ }^{838}$ und Belgratkapı wurde erst im Jahr 1886 wieder geöffnet. ${ }^{839}$ Basiert die Anzahl der Tore auf der Londoner Karte auf einem Irrtum des Kopisten, oder war irgendwann eines von den später zugemauerten Toren noch offen, was nur dem Kopisten der Vorlage bekannt gewesen sein konnte? In diesem Falle müßte diese Vorlage dann vor der Karte von Matrakçı Nasuh, also vor 1537 entstanden sein.

835 Diskussion dazu Berger, Langa Bostanı, 467, 475, Abb.4; Effenberger, Buondelmonti, 31.

836 Der Hafen ist in der Pariser Kopie als gerade Mauer wiedergegeben.

837 Yurdaydın, Matrakçı Nasuh, 8b.

838 Ahmet Muhtar Paşa, Kostantiniyye. Abgedruckt nach der ersten gedruckten Fassung aus dem Jahr 1900, İstanbul, Bedir Yayınevi, o. J.

839 Ebd., 71-72; vgl. Mehmet Ziya Bey, İstanbul ve Boğaziçi, 91. - Meyer-Plath/Schneider, 63 (Schneider) zufolge sei das Tor von 1189 bis 1886 zugemauert gewesen. Es muß um 1346 für eine Weile wieder geöffnet worden sein, siehe die Quelle bei Berger, Patria, 622, Anm. 98. - Vgl. auch Anm. 320 und 350. 
Hier soll zunächst auf einen spätosmanischen Bericht vom Ende des 19./ Anfang des 20. Jahrhundert hingewiesen werden. Ahmet Muhtar Paşa gibt im Zusammenhang mit der Öffnung des Belgratkapı im Jahr 1886 folgende Informationen ${ }^{840}$

Man nannte es ,Zugemauertes Tor', da es bis in neuere Zeiten zugemauert war. Es wurde aber wegen des griechischen Krankenhauses, das gegenüber dem Tor steht, während des letzten russischen Krieges wieder aufgemacht. Es wird wegen des früher hinter diesem Tor befindlichen Belgrat-Quartier (Belgrat Mahallesi) mit diesem Namen [Belgratkap1] genannt ... Vor dem Belgrat-Tor, oberhalb des Grabens befindet sich eine Grabenbrücke, die aus großen Quadern erbaut ist, hat aber ihren früheren Zustand gut bewahrt.

Obwohl in neuerer Zeit über die Brücke eine moderne Straße gelegt wurde, ist die osmanische Substanz noch deutlich zu erkennen (Abb. 65). Die Brücke läßt sich mit den anderen osmanischen Grabenbrücken, wie z.B. derjenigen des Silivrikapı (Abb. 12) vergleichen. Die Brücke war eindeutig vor 1886 noch vorhanden, wie auch aus Dethiers Aussage hervorgeht, doch kann sie nicht, wie dieser behauptete, aus der Zeit Süleymans des Gesetzgebers stammen, da das Tor damals vermauert war. ${ }^{841}$ Es handelt sich also um eine gleichzeitig mit den anderen entstandene und möglicherweise bereits im 15./16. Jahrhundert errichtete Brücke. Warum also sollten die Osmanen vor einem zugesperrten Tor noch eine Brücke erbaut haben? Es ist damit eindeutig erwiesen, daß das Tor seit der Eroberung bis spätestens ins 16. Jahrhundert weiterhin passierbar war. Das namenlose sechste Tor auf der Londoner Karte ist nur damit zu erklären, daß der Kopist eine ältere Vorlage benutzt hat, auf der sechs offene Tore ohne Beischriften wiedergegeben waren. Er hat jedoch die aktuellen Tornamen beginnend im Süden (oben) - eingetragen, weshalb das letzte (nördlichste) Tor ohne Namen blieb. Demzufolge plazierte er die Mihrimah Moschee nicht innerhalb dieses letzten Tors (das eigentliche Edirnekap1), sondern ein Tor davor, da er es unzutreffend als Edirnekapı beschriftet hatte.

Dieser Fehler kommt auf der Berliner Karte nicht vor. Dort besteht zwischen Yedikule und Silivrikapı ein großer Abstand ohne Angabe einer Toröffnung. Der Kopist der Berliner Karte wußte, daß seine Vorlage nicht dem tatsächlichen Zustand entspricht und an der Landmauer nur fünf Tore passiert werden konnten, Belgratkapı also bereits versperrt war. Es scheint, daß beide Kopisten eine Karte aus dem 15. Jahrhundert als Vorlage benutzt haben, wobei die Berliner Karte die Zahl der Tore korrigiert und die Mihrimah Moschee an ihrem richtigen Platz wiedergibt.

Die Londoner Kopie bildet als spätestes datiertes Monument die Yeni Valide Camii ab. Diese Karte wurde von Soucek mit überzeugenden Argumenten um

840 Ahmet Muhtar Paşa, Kostantiniyye, 71. - Vgl. Anm. 320 und 350.

841 Dethier, Bosphor, 52. 
1669 datiert. ${ }^{842}$ Wie oben erwähnt, wurde für die Berliner Karte eine Datierung zwischen 1663 und 1707/22 vorgeschlagen, wobei jedoch ein wichtiges Datierungskriterium unbeachtet blieb: Wie auf der Londoner wurde auch auf der Berliner Karte Tekfur Sarayı am nördlichsten Punkt der Theodosianischen Landmauer richtig wiedergegeben. Die Berliner Karte unterscheidet sich aber von der Londoner dadurch, daß hier unterhalb der Beischrift, die als „Saray-1 Tekur" gelesen werden kann, eine zweite Beischrift eingetragen ist, die das Gebäude als „Kâşıhâne“ (Fliesenwerkstatt) bezeichnet. Der Kopist teilt also die aktuelle Nutzung von Tekfur Sarayı mit. Anhand der Quellen ist zu belegen, daß Sultan Ahmet III. im Jahre 1719 beschlossen hatte, innerhalb dieses Gebäudes eine Fliesenwerkstatt einzurichten, wo Meister aus İznik Fliesen produzieren sollten. ${ }^{843}$ Die Werkstatt wurde 1724/25 eröffnet. Orbay ging auf diese Werkstatt kurz ein, hält aber eine Datierung der Karte um 1724/25 nicht für möglich, da diese mit dem von ihr angenommene Terminus nicht zu vereinbaren wäre. ${ }^{844}$

Weder die Darstellung der Arkadius-Säule noch eines seit 1722 nicht mehr existierendes İmaret liefern m. E. handfeste Indizien für eine Datierung. Die Wiedergabe eines bereits abgetragenen bzw. zerstörten Denkmals kann höchstens auf eine benutzte ältere Vorlage hinweisen. Auch das Fehlen eines Monuments wie der Çorlulu Ali Paşa Moschee kann nicht als Kriterium für einen terminus ante quem angesehen werden, da die Datierung einer historischen Karte nicht durch die Fehlende, sondern nur durch die dargestellten Monumente erfolgen kann, die einen sicheren terminus post quem markieren. Die İstanbulKarte von Piri Reis im Berliner Kitâb-1 Bahriye kann daher erst nach 1724/25 entstanden sein. Das Buch sollte somit in das zweite Viertel des 18. Jahrhunderts datiert werden.

Anhand der oben diskutierten Punkte ist mit einer gewissen Sicherheit zu behaupten, daß sowohl die Berliner als auch die Londoner Kopie eine Vorlage aus dem 15. Jahrhundert benutzt und sie zum größten Teil aktualisiert haben. Während schon die nicht aktualisierte Wiedergabe des Theodosius-Hafens auf beiden Karten einen eindeutigen Beleg für die Datierung der Vorlage liefert, bestätigt die Darstellung der Gartenanlage mit Umfassungsmauer zwischen Marmara-Seemauer und Yedikule sowohl die Datierung dieser früheren Karte als auch das Vorhandensein dieses ummauerten Areals im 15. Jahrhundert. Besonders die Londoner Karte liefert in der Gegenüberstellung mit dem archäologischen Befund den eindeutigen Hinweis, daß Belgratkapı nach der osmanischen Eroberung nicht ununterbrochen zugemauert, sondern im 15. Jahrhundert noch offen war. Die Vorlage dieser Karte muß demnach zwi-

842 Soucek, Mapmaking, 108-110.

843 Ahmet Refik, 65, Nr. 90.

844 Orbay, Ottoman Maps, 268, Anm. 342. 
schen 1453 und 1490 entstanden sein. Wie aber anhand der BuondelmontiKopien gezeigt werden konnte, muß das Xylokerkos-Tor (Belgratkapı) in spätbyzantinischer Zeit verschlossen gewesen sein (siehe Exkurs I). 